\title{
Les notes de Proust, une écriture dialogique
}

L'exemple du Cahier 46

Julie André

\section{(2) OpenEdition}

Journals

Édition électronique

URL : http://journals.openedition.org/genesis/634

DOI : 10.4000/genesis. 634

ISSN : 2268-1590

Éditeur :

Presses universitaires de Paris Sorbonne (PUPS), Société internationale de génétique artistique littéraire et scientifique (SIGALES)

Édition imprimée

Date de publication : 30 octobre 2011

Pagination : 157-172

ISBN : 978-2-84050-804-5

ISSN : 1167-5101

\section{Référence électronique}

Julie André, «Les notes de Proust, une écriture dialogique », Genesis [En ligne], 33 | 2011, mis en ligne le 23 octobre 2013, consulté le 30 avril 2019. URL : http://journals.openedition.org/genesis/634 ; DOI : $10.4000 /$ genesis. 634 


\title{
Les notes de Proust, une écriture dialogique L'exemple du Cahier 46
}

\author{
Julie André
}

$\mathrm{P}$ oust écrit dans le Cahier $57\left(f^{\circ} 42 v^{\circ}\right)$ : « Noter ne signifie rien si l'on veut bien songer que les ehoses réalités ne nous apparaissent jamais que sous des apparences particulières dont d'où il faudra les dégager. » Il refuse la littérature de notation, celle qui particularise et celle qui oblige à aller «prendre des notes 1 » sur le motif. Pour lui, le travail de l'écriture commence à partir du moment où l'on s'extrait de l'observation pour faire apparaître la réalité et la reconstruire. Et en effet on ne lui connaît pas, à proprement parler, de notes semblables à celles de Zola ou Flaubert et recueillies sur le terrain ${ }^{2}$. Si Proust tient bien des «carnets », ses notes ne sont que très rarement purement documentaires ${ }^{3}$, y figurent plutôt des fragments de vie réelle, des recherches onomastiques, des récits de rêves, des remarques sur des œuvres littéraires ou des idées d'abord pour le futur roman, puis pour le roman en cours. Seuls les tics de langage et les expressions verbales contenus dans les carnets pourraient s'apparenter aux notes prises par Zola et Flaubert puisqu'on peut penser qu'aussitôt entendues, elles sont ajoutées dans les carnets comme réserves d'expressions pour le roman ${ }^{4}$. À ces rares «notations», se mêlent les « annotations », courts commentaires sur ce qui a déjà été écrit ou ce qui reste à écrire.

Dans les cahiers de brouillon de Proust, on retrouve aussi ces deux types de notes, avec cependant une prédilection marquée pour $1^{\text {' }}$ " annotation ${ }^{5}$ » qu'on appelle également note de régie et qui consigne les réflexions de l'auteur sur son propre écrit, directives ou instructions destinées à préparer l'œuvre future. Ces notes apparaissent parfois dans les marges ou interrompent le fil d'une phrase, mais le plus souvent elles surgissent sur les versos. Une étude statistique des fonctions des versos dans un des cahiers de brouillon de Proust, le Cahier 46, m'a permis en effet de mettre en évidence que $25 \%$ des versos sont consacrés aux notes (pour seulement
$20 \%$ aux additions $)^{6}$; les marges et les paperoles étant plus régulièrement occupées par des " ajoutages ", parfois cependant introduits par une brève note. Une des particularités du brouillon proustien est en effet cette abondance de notes qui se répondent et s'organisent en réseau à l'intérieur des cahiers. Elles ne posent pas seulement les jalons de l'œuvre à venir, elles témoignent également des réflexions d'un écrivain sur l'œuvre en train de s'écrire et suggèrent, au sein de la fiction, la présence d'une voix : celle de l'auteur.

On a souvent souligné depuis les travaux de Mikhail Bakhtine et de Julia Kristeva la multiplicité des « voix » qui se font entendre dans le genre romanesque, qu'elles

1. À la recherche du temps perdu, édition publiée sous la direction de Jean-Yves Tadié, Paris, Gallimard, coll. « Bibliothèque de la Pléiade », 1987-1989, vol. III, p. 147.

2. Pour Zola, voir la présentation de Henri Mitterand, Carnets d' enquêtes, Paris, Plon, 1993. Pour Flaubert, voir l'édition de ses carnets par PierreMarc de Biasi dans Carnets de travail, Paris, Balland, 1988.

3. Comme le souligne Eugène Nicole, les recherches documentaires de Proust passent souvent par la correspondance. Il cite par exemple les lettres de Proust qui demande à son interlocuteur des renseignements sur les papiers japonais ou sur les robes de Fortuny. Eugène Nicole, «L'auteur dans ses brouillons : marginalia des Cahiers de Proust », Bulletin Marcel Proust, $\mathrm{n}^{\circ} 39,1989$, p. 61.

4. Voir l'article d'Antoine Compagnon sur les carnets, « Disproportion de Proust : les carnets de la Recherche », dans Carnets des écrivains, I, Paris, CNRS Éditions, coll. « Textes et Manuscrits», 1990, p. 164. Il remarque que « les fragments des carnets sont exceptionnellement des notations simples».

5. C'est à ces dernières que sera consacré cet article. Pour les «notations », voir notamment l'article d'Antoine Compagnon, art. cité, et le chapitre V « Tableaux vivants dans le roman », de Proust entre deux siècles, Paris, Éditions du Seuil, 1989, p. 127-152.

6. En effet, les autres versos sont blancs $(29 \%)$ ou consacrés à la rédaction $(26 \%)$. Voir Julie André, « Le Cahier 46 de Marcel Proust. Transcription et interprétation ", thèse de doctorat sous la direction de Pierre-Louis Rey, Université de Paris III, 2009, vol. I, p. 54. 
soient celles de l'écrivain, du destinataire ou du contexte culturel 7 et on sait que ce dialogisme est particulièrement marqué dans le roman moderne. Cependant, il semble que cette dimension « ambiguë8 », « autocritique 9 » pour reprendre les termes de Julia Kristeva et de Bakhtine, soit redoublée dans les brouillons de Proust qui mettent en scène un double dialogue : le dialogue intérieur de l'auteur d'abord, en révélant les interrogations incessantes de l'auteur sur son propre texte et ensuite, le dialogue interne à la page, entre l'écriture marginale et le récit, entre ce qui est écrit et ce qui reste à écrire. En prenant l'exemple du Cahier 46, que Proust a rédigé au cours des années 1914 et 1915 et qui me semble exemplaire de sa pratique du brouillon ${ }^{10}$, c'est aux notes de Proust - comme lieu du croisement des différentes voix - et à leur langage que je voudrais m'intéresser. Car c'est peut-être de ce double dialogue tel qu'il apparaît dans les brouillons de Proust - dialogue entre la note et le texte, dialogue entre l'auteur-relecteur et l'auteur-scripteur - que naît la richesse d'une écriture qui, l'œuvre une fois achevée, jouera sur les instances narratives et ne séparera pas le propos sur l'œuvre de l'œuvre elle-même.

\section{Espace et langage}

Plusieurs articles sont consacrés aux notes dans les brouillons de Proust ${ }^{11}$, en particulier celui d'Anne Herschberg Pierrot qui les définit comme un « langage second sur du langage 12 » et souligne leur absence d'autonomie référentielle. À partir de son étude du Cahier 57, elle précise ce qui caractérise en premier lieu les notes de Proust :

D'abord leur place, aux bords de la version rédigée, en marge et sur les versos du cahier. Mais la marginalité n'est pas le signe d'une moindre importance. Au contraire, ce sont les notes ajoutées, et l'urgence de dire, soulignée sans arrêt par les «Faute de place je mets ici », qui prennent le devant de la scène de l'écriture ${ }^{13}$.

Si la position de la note dans l'espace de la page est bien la même dans le Cahier 46, il semble que le manque de place et que l'urgence à dire prennent une place beaucoup moins importante que dans le cahier étudié par Anne Herschberg Pierrot. Dans notre Cahier, qui a pour objet d'introduire Albertine dans le roman, la limitation de l'espace apparaît dans la graphie à travers la taille des caractères qui deviennent parfois minuscules $\left(\mathrm{f}^{\circ} 47 \mathrm{v}^{\circ}\right)$, à travers la sursaturation de certaines pages (fos $61 \mathrm{v}^{\circ}$ et $62 \mathrm{r}^{\circ}$, voir fig. 1) ou encore par les renvois à d'autres folios (« ce que j'ai barré est au recto en face », $\mathrm{f}^{\circ} 48 \mathrm{r}^{\circ}$ ) mais aussi à travers la fréquence des «ne pas oublier» (fos $52.2 \mathrm{r}^{\circ} 14,62 \mathrm{r}^{\circ}$, voir fig. 1, et $94 \mathrm{v}^{\circ}$ ) ou « penser à » (f॰ $95 \mathrm{v}^{\circ}$, voir fig. 2) ou enfin, dans le tracé même des lettres qui témoignent de la vitesse de la plume sur le papier. Pourtant, contrairement à ce qui advient dans le Cahier 57, cet aspect n'est que rarement noté par l'écrivain : seule la paperole du folio 52.2 précise qu'un

7. Julia Kristeva, «Le mot, le dialogue et le roman », Sémiotikè. Recherches pour une sémanalyse, Paris, Éditions du Seuil, 1969 (1966), p. 144. Elle cite d'ailleurs l'exemple de Proust à la page 152.

8. Julia Kristeva, Le Temps sensible, Paris, Gallimard, « nrf Essais », 1994, p. 388 .

9. Voir la présentation des travaux de Mikhaïl Bakhtine par Tzvetan Todorov, Mikhaïl Bakhtine. Le principe dialogique, Paris, Éditions du Seuil, 1981, p. 103.

10. En effet, ce cahier est situé dans l'entre-deux, entre les premiers brouillons dans lesquels les notes sont rares et les versos d'une manière générale peu utilisés (voir par exemple, les cahiers « Sainte Beuve », Cahiers 6 et 7), et les cahiers postérieurs aux versos le plus souvent saturés (voir le Cahier 57 cité plus loin qui date de 1911 mais contient de nombreuses notes et additions postérieures).

11. Antoine Compagnon s'est intéressé à cette pratique dans les carnets (art. cité), Eugène Nicole, à l'écriture marginale dans les brouillons de Proust (art. cité), Florence Callu au célèbre « capital, capitalissime » qui ponctue si souvent les notes de Proust. Voir Florence Callu, «"Capital, capitalissime", un mode de composition chez Marcel Proust ? », dans Leçons d'écriture. Ce que disent les manuscrits. Hommage à Louis Hay, textes réunis par Almuth Grésillon et Michael Werner, Paris, Lettres modernes Minard, 1985, p. 79-89.

12. Voir « Les notes de Proust », Genesis, « Enjeux critiques », n 6, 1994, p. 61.

13. Ibid., p. 67.

14. Ce folio, tombé ou arraché du Cahier 46, a été retrouvé par Chizu Nakano dans un Carton en 1989. Voir « Un reliquat inédit pour l'histoire d'Albertine », Bulletin d'Informations proustiennes, $\mathrm{n}^{\circ} 21,1990$, p. 75-82. J'ai montré dans ma thèse qu'il fallait replacer ce folio surchargé de paperoles après le folio 52 et avant le folio 53. Voir Julie André, « Le Cahier 46 : transcription et interprétation », thèse citée, p. 89. 
morceau a été « collé faute de place15 ». En revanche, l'importance de ce qui doit être écrit - l'importance des ajouts - est sans cesse soulignée. Les traces de cette valeur accordée aux additions apparaissent dans la graphie des notes qui les ouvrent avec l'emploi de capitales ( AMOUR », $\mathrm{f}^{\circ} 83 \mathrm{r}^{\circ}$ ) ou de caractères plus gros pour signaler l'entrée dans un nouveau chapitre : « Je commence ici (en face tout ce qui concerne Albertine depuis Ba[lbec] le chapitre : Jetnes À l'ombre des Jeunes filles en fleurs) » $\left(f^{\circ} 46 v^{\circ}\right)$. Fragments plutôt que textes, les notes se signalent ainsi parfois comme telles du simple fait de leur graphisme ou de leur disposition sur la page ${ }^{16}$.

Au-delà de sa position à la marge du texte, sur les versos ou les paperoles, et de sa forme, le plus souvent îlot noir dans le blanc de la page, la note s'annonce également comme métalinguistique par d'autres indices non verbaux. Ainsi, la note s'immisce parfois au cœur même du texte par l'usage des parenthèses. Au folio $95 \mathrm{v}^{\circ}$, lorsqu'il est question des relations entre Saint-Loup et Albertine et du fait que « un peu plus tard » «St Loup vint souvent [...] dire bonjour à la station de Doncières », une note, sans autre signe de rupture avec le fil de la narration que la présence de parenthèses, précise seulement :

(penser à la mettre à son moment dans les retours Verdurin) (Cahier 46, $\mathrm{f}^{\circ} 95 \mathrm{v}^{\circ}$, voir fig. 2).

Ici la valeur notative de la phrase se déduit de l'emploi de parenthèses qui marquent l'isolement et le détachement de celle-ci par rapport au texte, mais pas seulement. La rupture se manifeste également dans le style elliptique propre à la note : emploi d'un verbe à l'infinitif, ellipse du déterminant. Tout comme les parenthèses, les termes qui ouvrent les notes ${ }^{17}$ et qui, par là, les signalent, fonctionnent comme des indications de régie. Ces termes sont le plus souvent des infinitifs injonctifs - « ajouter », « dire », « ne pas oublier », « penser à 18 »- ou des verbes impersonnels - « il faudra 19 ». On relève également d'autres mots qui annoncent la note, et en particulier le mot « note » lui-même qui, quoiqu'il n'apparaisse jamais en tant que tel dans le Cahier $46^{20}$, surgit sous sa forme latine : "Nota Bene » (fo $47 \mathrm{v}^{\circ}$ ), parfois abrégé en «N. B. » (fos $52 \mathrm{v}^{\circ}, 62 \mathrm{v}^{\circ}$, voir fig. 7). Ce n'est donc pas seulement et pas toujours la disposition qui signale la note proustienne mais bien plutôt son élocution. Plus qu'un espace, la note est un langage.

\section{« Kapital $»:$ le « lansgage » de Proust}

Comme l'a souligné Anne Herschberg Pierrot, un mot ouvre régulièrement les notes de Proust : le fameux « capital ». Dans le Cahier 46, le mot et ses différents dérivés ${ }^{21}$ sont relativement nombreux, une vingtaine, moins toutefois que dans le Cahier 57 qui est plus ancien mais contient de nombreuses additions postérieures 22 pour Le Temps retrouvé et plus que dans le Cahier 71 par exemple23. Le Cahier 46 qui date de 1914-1915 appartient à cet espace d'entre-deux, avant que l'urgence à dire ne devienne une préoccupation constante de l'écrivain à l'approche de la mort, mais après qu'il a commencé le grand roman. Ajouté sur les versos, ou parfois en marge 24 , le mot «capital » témoigne toujours d'une relecture du scripteur qui désigne par ce terme les passages à conserver ou, plus souvent, signale les ajouts à intégrer au texte du brouillon. Mais il semble que Proust fasse du terme

15. Dans le Cahier 54, on relève également cette expression. Voir, par exemple, les folios $18 \mathrm{r}^{\circ}$ et $21 \mathrm{r}^{\circ}$, Cahier 54 -Cahiers 1 à 75 de la Bibliothèque nationale de France, introduction et analyse de Nathalie Mauriac Dyer et transcription, notes et index de Francine Goujon, Nathalie Mauriac Dyer et Chizu Nakano, Turnhout (Belgique), BnF/Brepols, 2008, 2 vol.

16. On pourrait y ajouter l'utilisation de la couleur pour souligner ou mettre en relief. À la page «Amour», le mot est écrit en rouge, par exemple.

17. Anne Herschberg Pierrot a montré que les additions commençaient fréquemment par «d'ailleurs ». Dans notre cahier, on en trouve sur la paperole du folio $51 \mathrm{v}^{\circ}$, la paperole du folio $52.2 \mathrm{r}^{\circ}$ et sur les folios $80 \mathrm{v}^{\circ}$ et $81 \mathrm{r}^{\circ}$ (fig. 5), et un bel exemple au folio $61 \mathrm{v}^{\circ}$ (fig. 1). Voir Pierre-Marc de Biasi, Anne Herschberg Pierrot et Jacques Neefs, « Ajout et genèse », Figures d'ajout : phrase, texte, écriture, Paris, Presses de la Sorbonne nouvelle, 2002, p. 38.

18. Pour des exemples d'infinitifs, voir les folios $57 \mathrm{r}^{\circ}, 62 \mathrm{v}^{\circ}$ (fig. 7), $94 \mathrm{v}^{\circ}$, $95 \mathrm{v}^{\circ}$.

19. Voir les folios $94 \mathrm{v}^{\circ}$ et $96 \mathrm{v}^{\circ}$ par exemple. Il faut noter que la première personne est rarement employée pour ouvrir une note.

20. Anne Herschberg Pierrot indique que le mot « note » apparaît une fois dans le Cahier 57. «Les notes de Proust », art. cité, p. 68.

21. Au folio $79 \mathrm{v}^{\circ}$ du Cahier, on trouve un « capitalissimum » non recensé par Florence Callu.

22. Les rectos dateraient de 1911, tandis que les versos auraient été écrits dans les années 1916-1917. Voir Anne Herschberg Pierrot, «Les notes de Proust », art. cité, p. 67.

23. Voir Florence Callu, art. cité, p. 87-88 et le tableau en annexe. Les cotes 16686 et 16697 appartiennent respectivement aux Cahiers 46 et 54 . 24. Pour un exemple, voir le folio $63 \mathrm{v}^{\circ}$. 


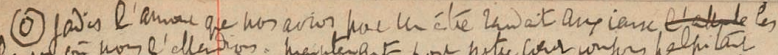

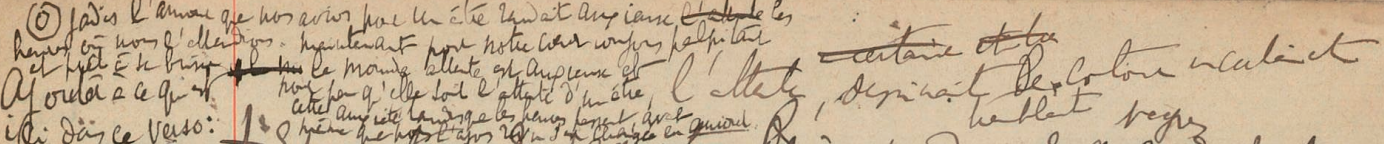

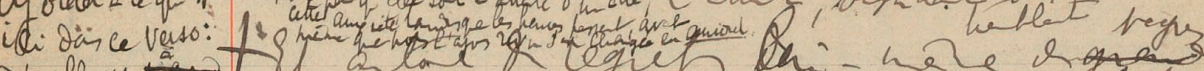

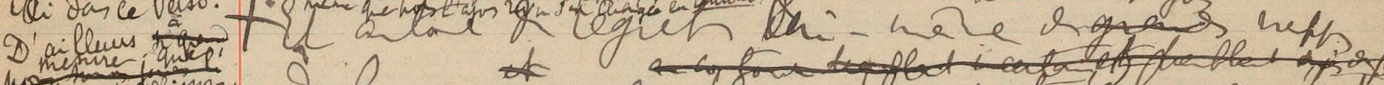

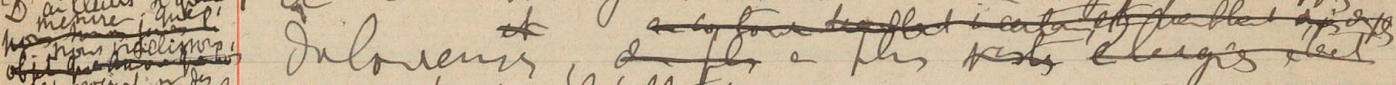

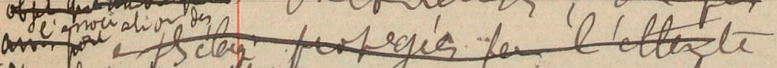

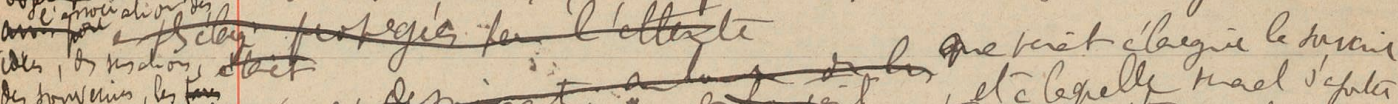

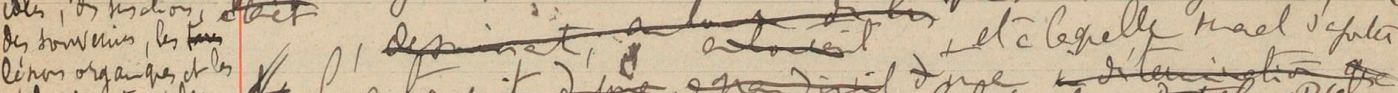

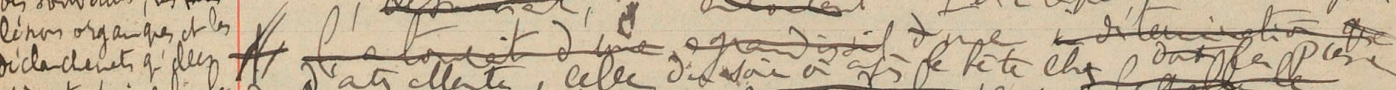

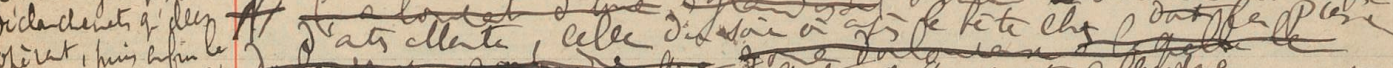

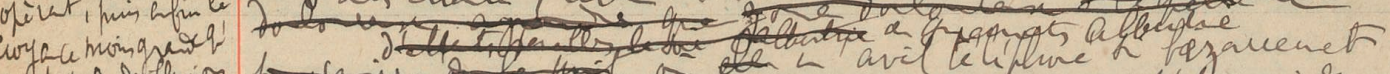

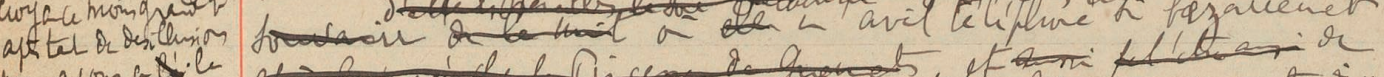

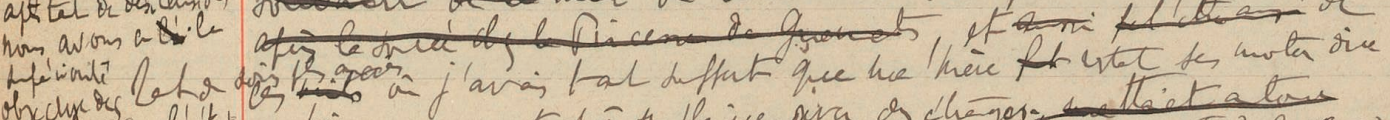

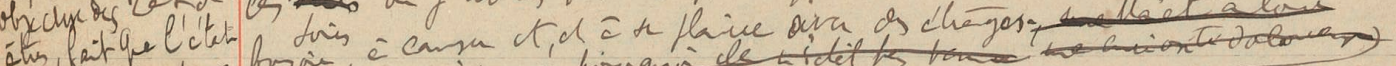

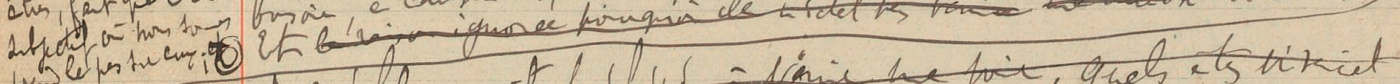
anis

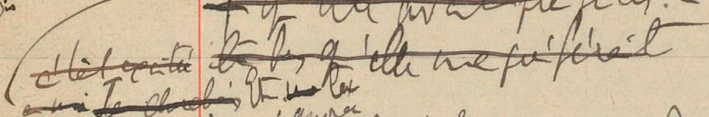

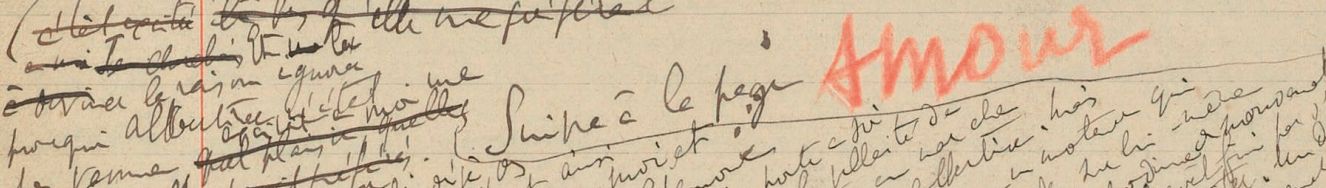

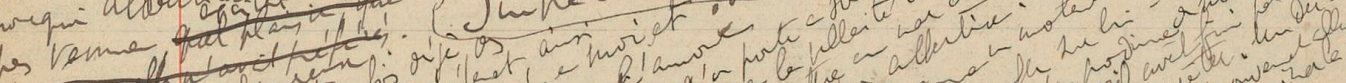

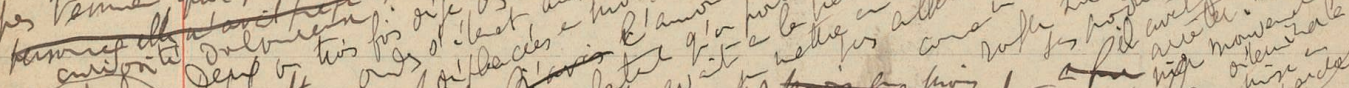

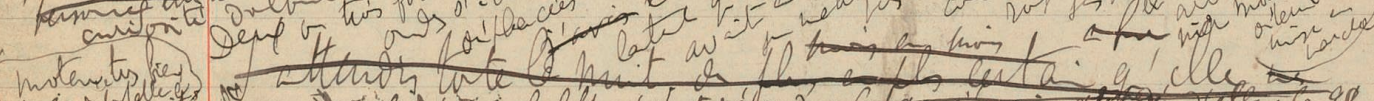

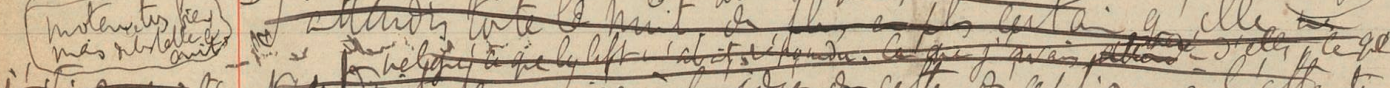

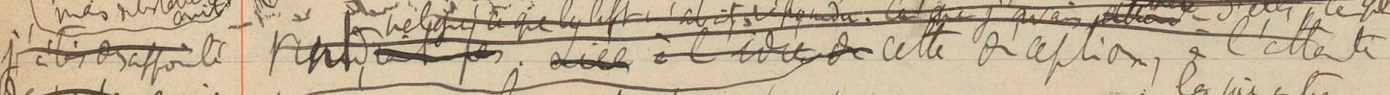

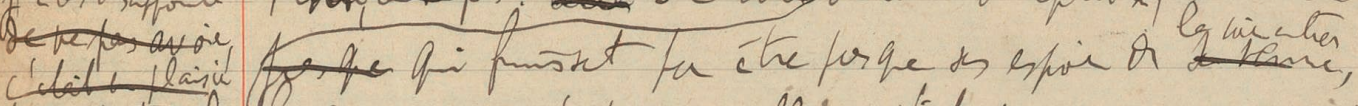
his a la ot tor $1+20$ the imfresion Dalmux.

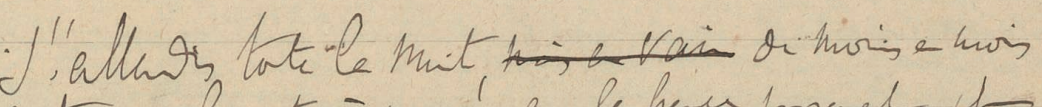

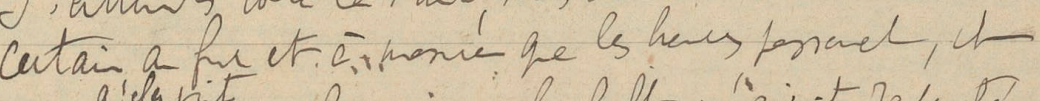

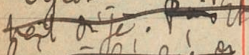

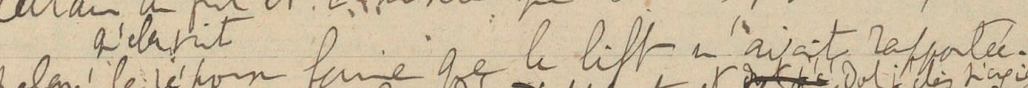

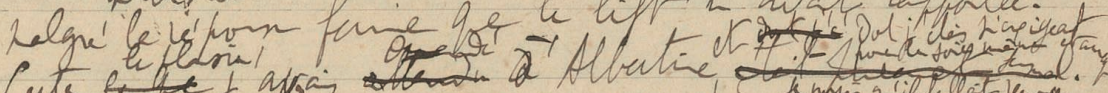

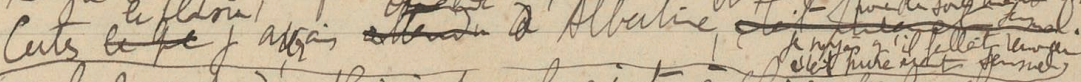

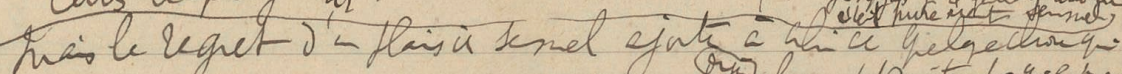

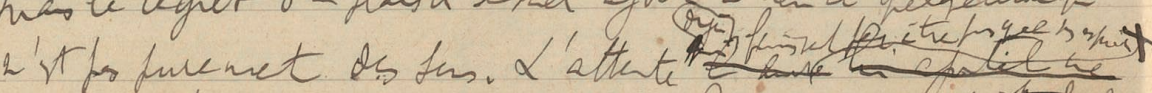

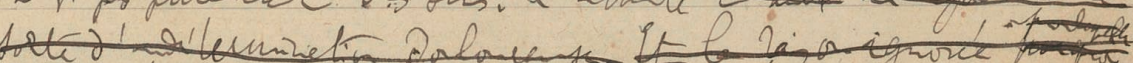

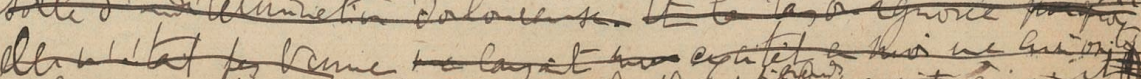

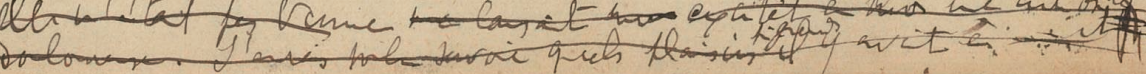

Fig. 1 : Marcel Proust, Cahier 46 (BnF, NAF 16686, fos $\left.61 v^{\circ}-62 r^{\circ}\right)$ 


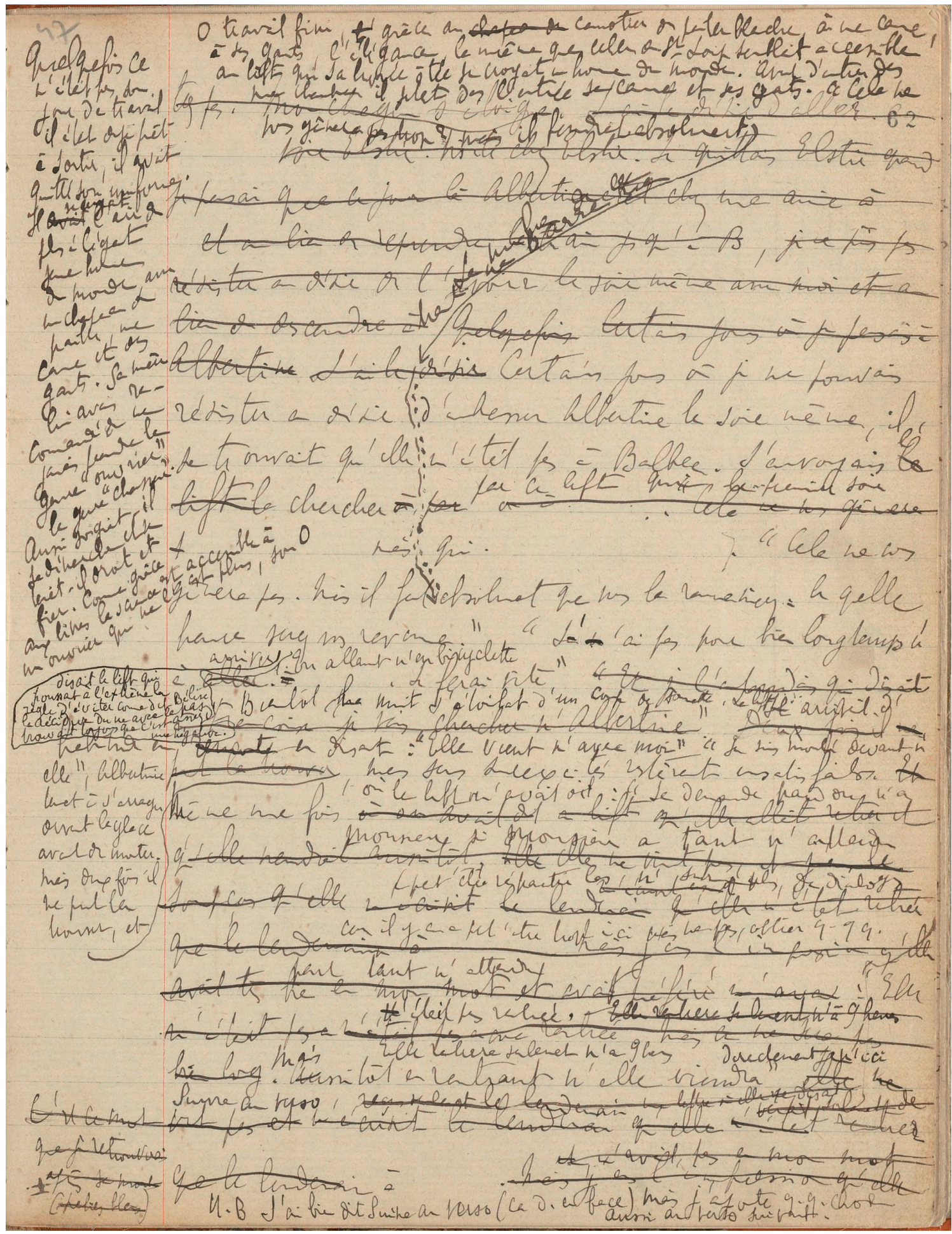




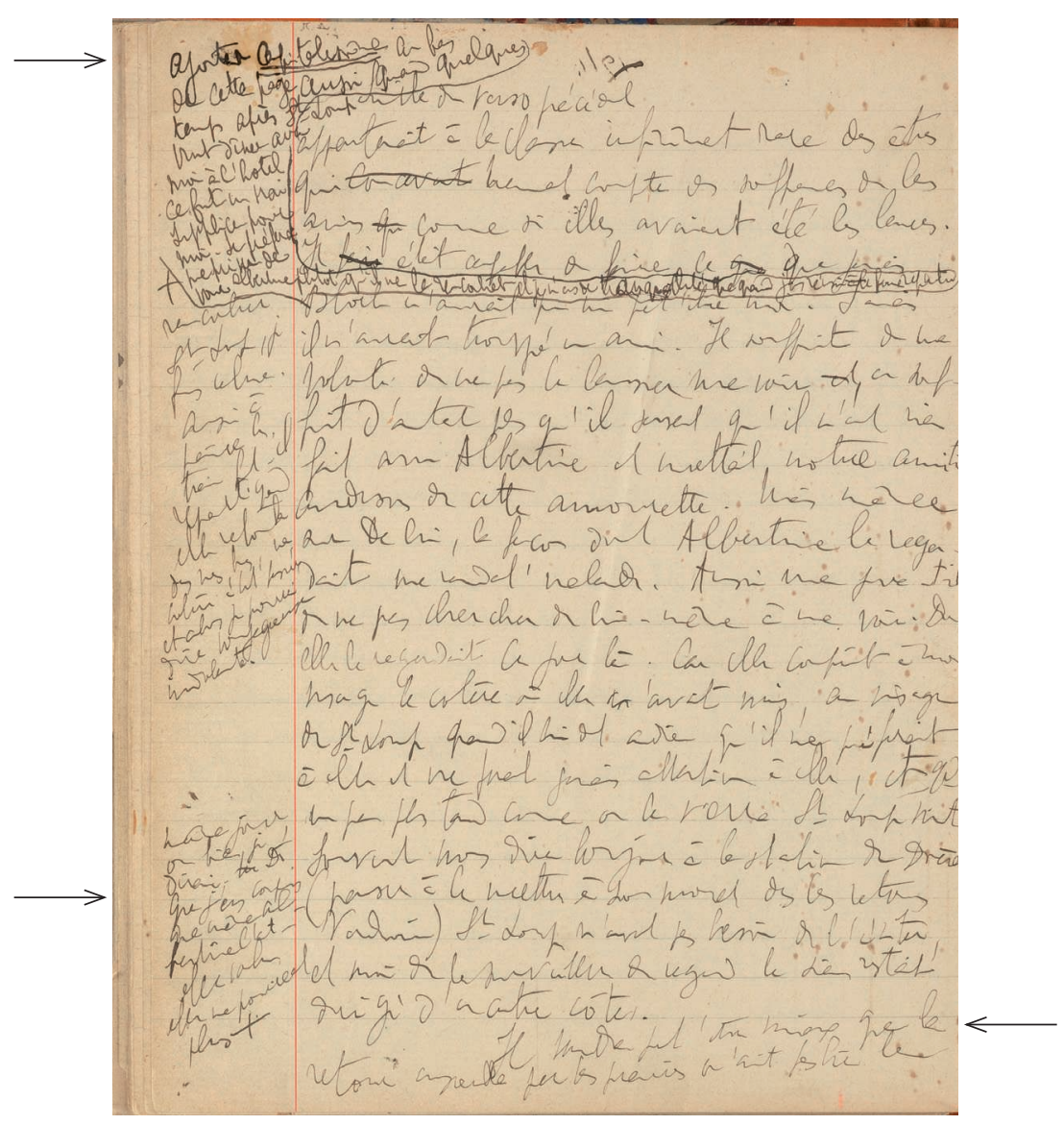

Fig. 2 : Marcel Proust, Cahier $46\left(\mathrm{BnF}, \mathrm{NAF} 16686 \mathrm{f}^{\circ} 95 \mathrm{v}^{\circ}\right)$

un usage très personnel. Florence Callu a noté les superlatifs ( capitalissime ») dont Proust affuble le mot. Dans le Cahier 46, « capitalissime » est presque aussi fréquent que « capital », et dans certains cahiers, le superlatif français est parfois redoublé d'un superlatif latin 25 , «capitalissimus 26 » ou «capitalissimum » (f $79 \mathrm{v}^{\circ}$, voir fig. 3) ce qui suggère l'hypothèse d'un sens fort du mot chez Proust. Il est également employé en renfort du soulignement pour indiquer un passage qui semble particulièrement important 27 ou encore écrit en lettres capitales ( $\mathrm{f}^{\circ} 53 \mathrm{v}^{\circ}$, voir fig. 4), parfois avec une telle intensité et une telle fréquence qu'il semble réactiver l'ancien sens de « qui peut coûter la tête, mortel28». De manière presque contradictoire, le mot conserve pourtant ailleurs la valeur sémantique habituelle puisqu' on le trouve, à plusieurs reprises, intégré dans une phrase :

Mais en tous cas le mettre q. q. part ici ou ailleurs car c'est capital (Cahier 46, fo $62 \mathrm{v}^{\circ}$, fig. 7). ou encore :

Cette addition de ce verso est capitale (Cahier 46, $\mathrm{f}^{\circ} 80 \mathrm{v}^{\circ}$,

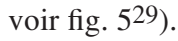

Le contexte même vient en nuancer l'importance. La paperole du folio $46 \mathrm{v}^{\circ}$, par exemple, s'ouvre sur les mots

25. C'est parfois aussi la répétition qui marque l'intensité, comme dans cette phrase du Cahier 57, dans lequel on trouve « Capitalissime issime, issime de peut'être le plus de tte l'œuvre : quand je parle du plaisir éternel de la cuiller, tasse de thé etc $=$ art $»\left(\right.$ verso paperole du $\left.\mathrm{f}^{\circ} 19\right)$. Voir Matinée chez la princesse de Guermantes. Cahiers du Temps retrouvé, édition critique établie par Henri Bonnet en collaboration avec Bernard Brun, Paris, Gallimard, 1982, p. 331.

26. Voir le tableau qui figure à la fin de l'article de Florence Callu, art. cité, p. $87-88$.

27. Dans les Cahiers 54 et 71 , on trouve plus régulièrement « important » ou « essentiel».

28. Alain Rey, Dictionnaire historique de la langue française, Paris, Le Robert, 1998, vol. I.

29. Voir aussi le folio $54 \mathrm{r}^{\circ}$ (fig. 4). 


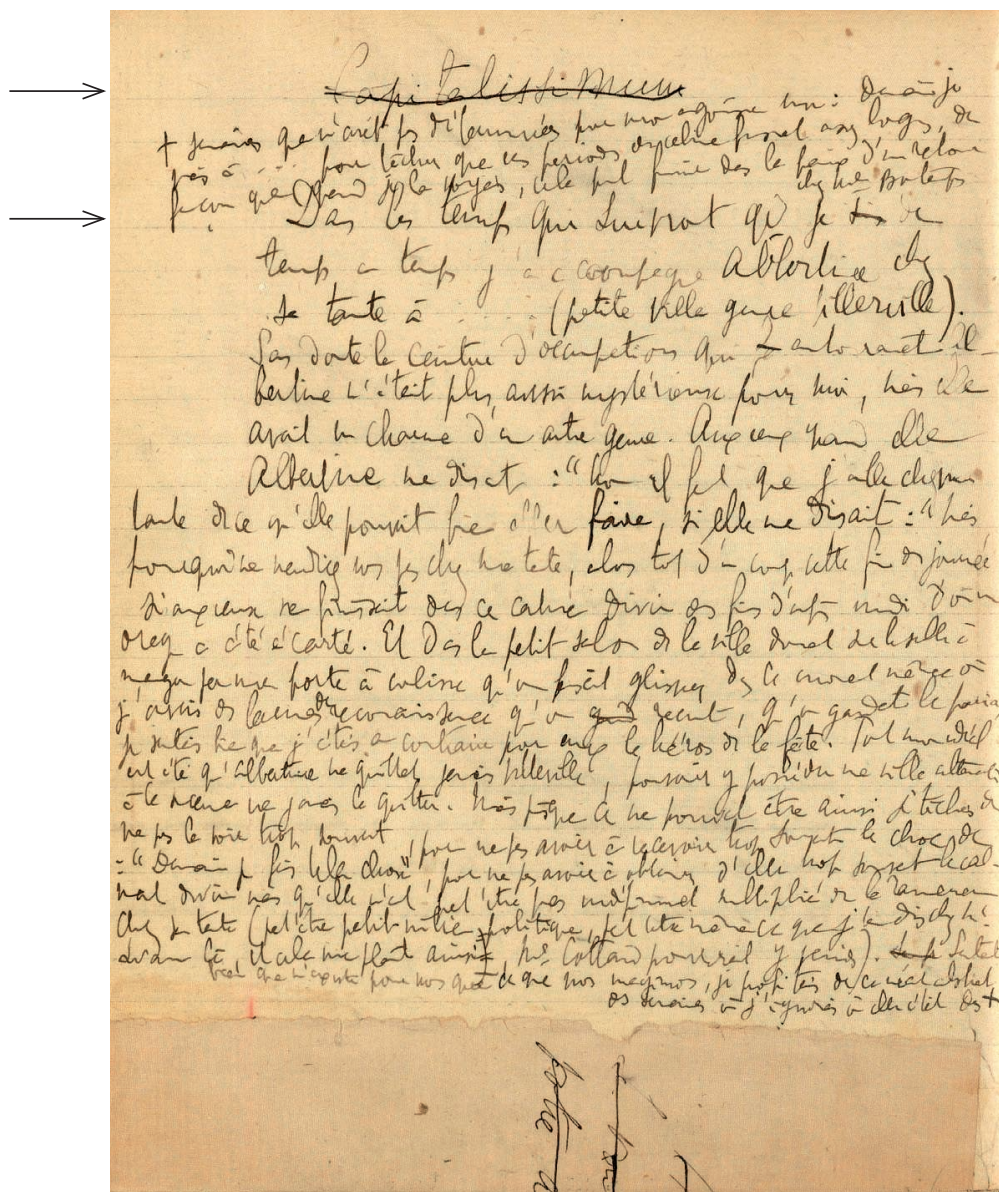

Fig. 3 : Marcel Proust, Cahier $46\left(\mathrm{BnF}, \mathrm{NAF} 16686, \mathrm{f}^{\circ} 79 \mathrm{v}^{\circ}\right)$
«Capitalissime à mettre là » mais se clôt sur une note qui, du fait de l'emploi du modalisateur « sans doute », sonne comme une rectification : «(Ce papier collé qui est sans doute beaucoup mieux que le reste pourra le remplacer ou s'y mêler) ». Plus remarquable encore, si, dans la première partie de ce cahier, le mot « capital» est accompagné de verbes ${ }^{30}$ comme « mettre », « dire » ou « ajouter », en revanche, dans la seconde, il tend à indiquer seul le début de la note 31 . Dès lors, on peut s'interroger sur la valeur du terme. A-t-il véritablement une « valeur d'icône ${ }^{32}$ » ? Il semble en effet, à la fin du Cahier, perdre cette fonction de soulignement et devenir un simple signal de la note. Le mot « capital » serait, dans ce cas, un simple marqueur du caractère notatif du passage ${ }^{33}$, par opposition au texte, à la rédaction, ce qui constituerait une autre explication de la multiplication de l'emploi de ce terme dans les cahiers d'après 1914 constatée par Florence Callu.

De cette appropriation du mot témoigne son orthographe : Proust l'écrit en effet parfois avec un K ( $f^{\circ} 53 \mathrm{v}^{\circ}$, fig. 4) ${ }^{34}$. Cette tendance à déformer le lexique, et en particulier l'orthographe et la prononciation des mots, n'est pas rare chez Proust. On la retrouve dans ce qu'il appelle lui-même «lansgage35 », langage intime qu'il utilise dans les lettres à ses amis proches et notamment à Reynaldo Hahn, à qui il envoie par

30. « Capitalissime à mettre là » (paperole du f $46 \mathrm{v}^{\circ}$ ), « Pour mettre quand elle est là et que je suis calmé (Capitalissime) » (fo $53 \mathrm{v}^{\circ}$, fig. 4), «Mettre après cette scène (capital) » $\left(\mathrm{f}^{\circ} 54 \mathrm{v}^{\circ}\right)$, « Mettre (capitalissime) quand elle m'a quitté » (paperole du $\left.\mathrm{f}^{\circ} 54 \mathrm{v}^{\circ}\right)$, «Dire aussi (capital) » $\left(f^{\circ} 57 r^{\circ}\right)$. On trouve seulement deux fois un infinitif suivi du mot « capital » dans la suite du cahier : «Ajouter Capital » (fo $62 v^{\circ}$, fig. 7, et $\left.76 v^{\circ}\right)$. 31. Folios $63 \mathrm{v}^{\circ}, 66 \mathrm{v}^{\circ}, 79 \mathrm{r}^{\circ}, 84 \mathrm{v}^{\circ}, 94 \mathrm{v}^{\circ}$.

32. Anne Herschberg Pierrot, «Les notes de Proust », art. cité, p. 68.

33. On pourrait ainsi l'envisager comme un simple équivalent du mot « note ».

34. Voir également le «Kapitalissime » du folio $67 v^{\circ}$. Florence Callu précise que les graphies sont «variables » et note l'existence de « Kapitalissime », « KKKapitalissime », « CCCapitalissime », art. cité, note 5 , p. 89.

35. Voir Martin Robitaille, Proust épistolier, Montréal, Presses de l'université de Montréal, coll. « Espace littéraire », 2003, p. 192 et les Notices biographiques des correspondants établies par Virginie Greene, dans Lettres, sélection et annotation de Françoise Leriche, Paris, Plon, 2004, p. 1240. 
$\vee$
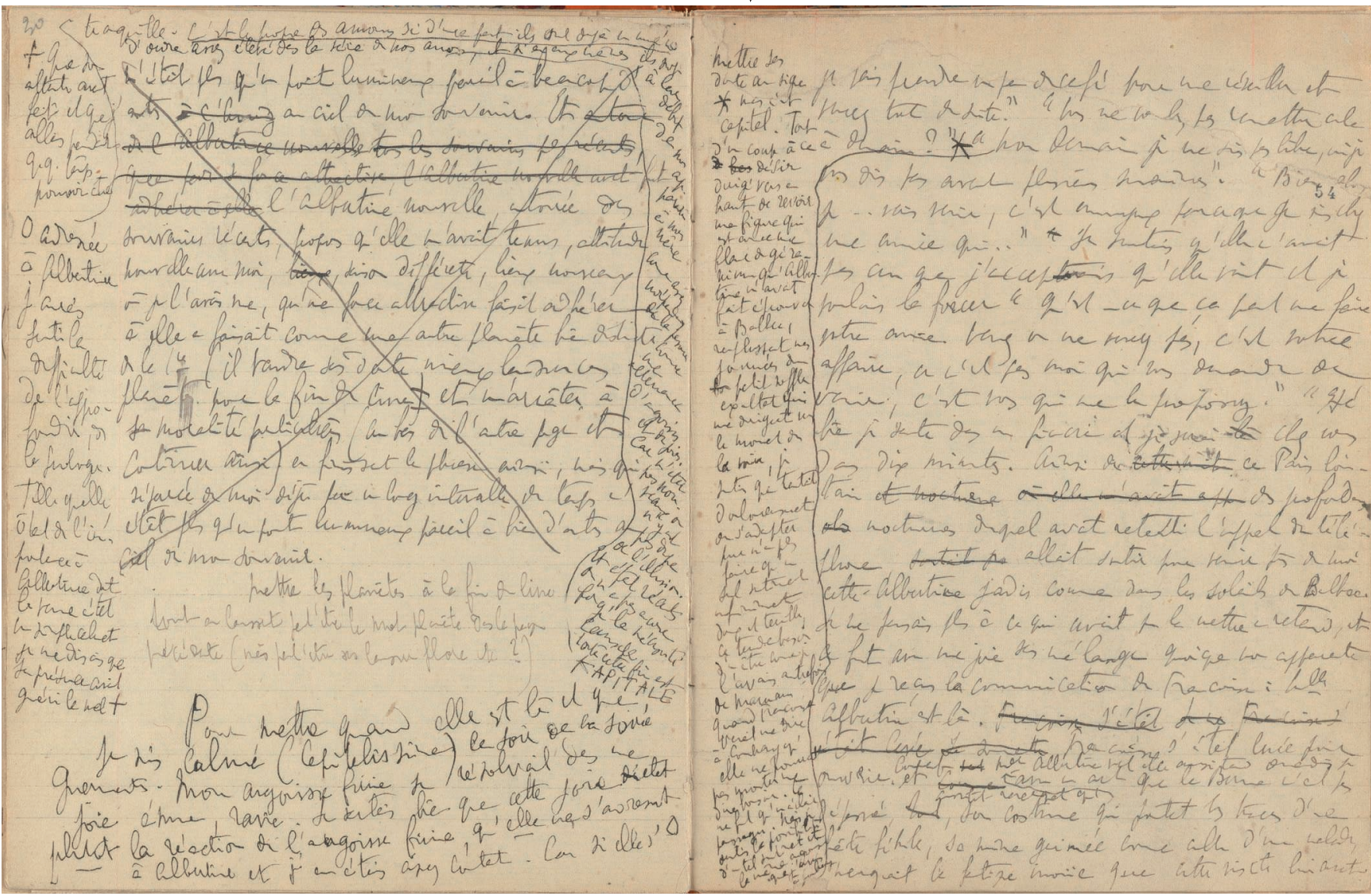

Fig. 4 : Marcel Proust, Cahier 46 (BnF, NAF 16686, fos $53 v^{\circ}-54 r^{\circ}$ )

exemple « Mille petites caresnuls, genstilib ». D'ailleurs, dans ses lettres, on remarque que la lettre $\mathrm{K}$ remplace souvent le C. Par exemple, «kouscher » est mis pour « coucher », « Karlilch » pour « Carlyle », « Kimetière » pour « cimetière 37 »; de même que le son [ch], l'usage de cette lettre $\mathrm{K}^{38}$ semble être un trait récurrent de cette personnalisation du langage. Le mot « Kapital », s'il sert à souligner, permet également de mettre en évidence une appropriation du langage de la note. Trace d'une écriture personnelle, intime ${ }^{39}$, ce langage de la note signale non plus un dialogue avec l'ami, mais avec soi-même et met l'accent sur la dimension dialogique de l'écriture au brouillon. Il porte la trace d'un dédoublement du je, reflet de « la distance de l'auteur à l'égard de lui-même ${ }^{40} »$.
36. Pour des exemples de ce « lansgage », voir, dans l'ouvrage de Martin Robitaille, la partie consacrée à la correspondance avec Reynaldo Hahn (p. 171-204) et, dans L'Eil de Proust, les dessins des lettres à Reynaldo Hahn. Philippe Sollers, L'Eil de Proust, Paris, Stock, 1999, p. 57-92. 37. Voir L'Eil de Proust, op. cit., p. 65 et Proust épistolier, op. cit., p. 199, 203.

38. Faut-il y voir également une trace de la guerre et de l'influence de la langue allemande ? Une lettre de 1915 pourrait accréditer cette thèse : Proust y orthographie « Kultur » et « Kolossal » avec un K. Lettres, op. cit., p. 727.

39. Virginie Greene souligne que le « lansgage » disparaît des lettres à Reynaldo Hahn à partir de la déclaration de guerre et de l'engagement de ce dernier (Lettres, op. cit., p. 1241) : n'est-ce pas précisément à ce moment-là qu'il apparaît dans les brouillons ?

40. Je reprends ici les mots de Julia Kristeva, art. cité, p. 155. 


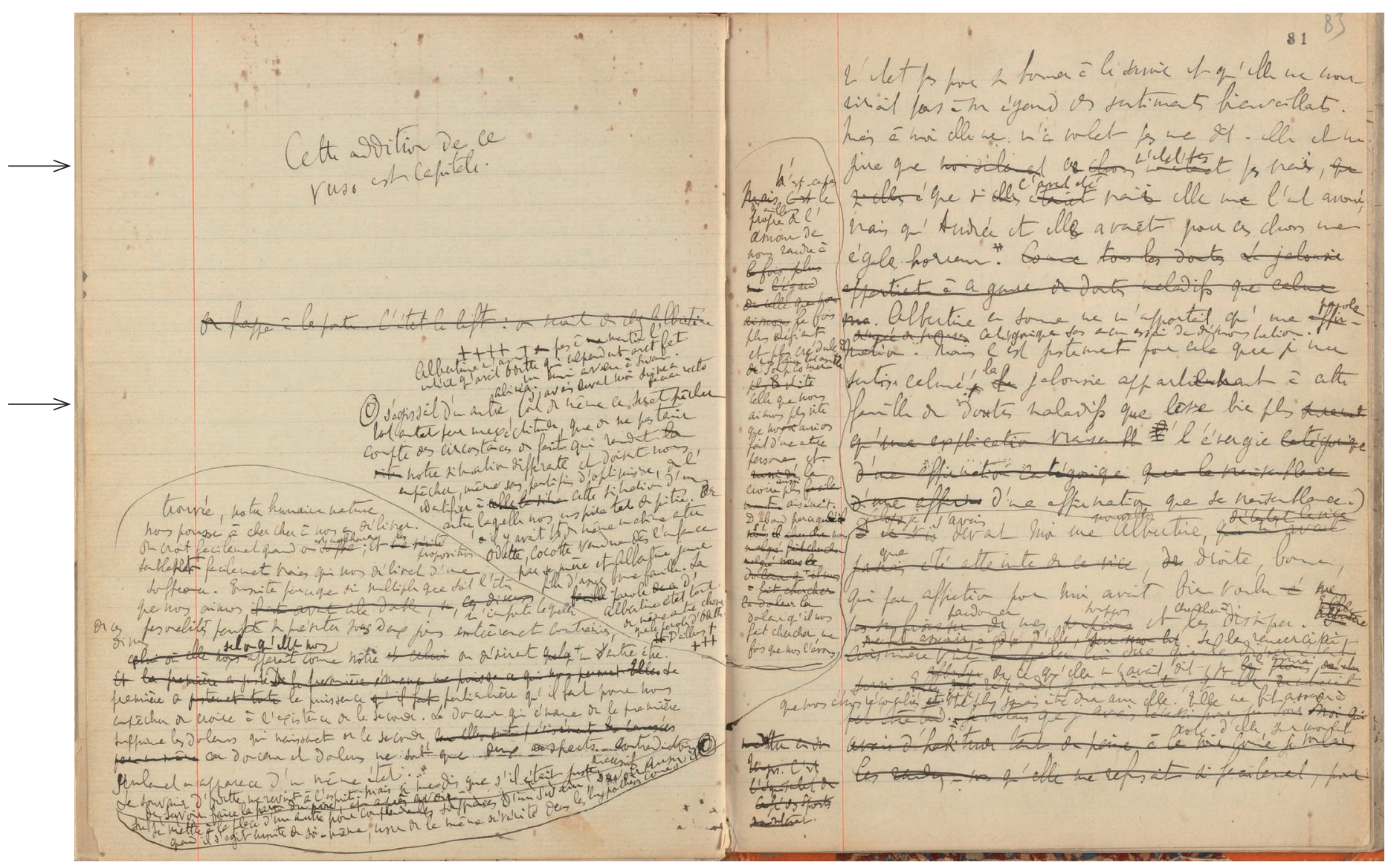

Fig. 5 : Marcel Proust, Cahier 46 (BnF, NAF 16686, fos $\left.80 v^{\circ}-81 r^{\circ}\right)$

\section{Modalisations}

D'emblée, la présence d'un dialogue dans les notes est perceptible par l'emploi de structures interrogatives :

N. B il vaudra mieux peut'être mettre le nez et les lèvres après les yeux ? (Cahier 46, $\left.\mathrm{f}^{\circ} 52 \mathrm{v}^{\circ}\right)$.

$\mathrm{Ou}$ :

Mettre les planètes à la fin du livre

tout en laissant peut'être le mot planète dans la page précédente (mais peut'être sans laisser flore etc ?) (Cahier 46, $f^{\circ} 53 \mathrm{v}^{\circ}$, voir fig. 4).
Dans les deux cas, on note cependant la présence de la locution adverbiale «peut-être » qui introduit le doute. Ces exemples témoignent des hésitations de l'écrivain sur ce qu'il vient d'écrire et d'un dialogue entre le texte et la note. Parfois même, le doute porte non pas sur la page écrite mais sur d'autres scènes du roman en cours :

\section{Peut'être}

faudrait-il que l'entrée dans l'

hôtel devant les glaces soit

d'Albertine au lieu de

$\mathrm{S}^{\mathrm{t}}$ Loup mais je

ne crois pas $\left(\right.$ Cahier $\left.46, \mathrm{f}^{\circ} 46 \mathrm{v}^{\circ}\right)$. 
Dans ce cas, le dialogue ne met pas en jeu les deux instances que sont l'auteur-scripteur et l'auteur-lecteur mais s'instaure au sein même de l'instance de l'auteurlecteur. Le texte de la note retrace ainsi l'évolution du roman et du processus psychique de la création en révélant les interrogations qui se font jour dans la pensée de l'écrivain. Il est d'ailleurs frappant de constater que ces diverses hypothèses sont parfois suivies de « réponses ». Ainsi, à la note marginale : «Peut'être pourrai-je mettre ma vie couchée, mes sensations de saison avant ceci et après le milieu Guermantes » succède une autre note : "Mais cela mettrait peut'être la soirée chez la Pcesse de Guermantes trop longtemps après ma connaissance des Guermantes " $\left(f^{\circ} 48 \mathrm{r}^{\circ}\right)$. Réponse qui apparaît parfois sous la simple forme d'un non : « non ceci vient avant » $\left(\mathrm{f}^{\circ} 84 \mathrm{r}^{\circ}\right)$. On remarque également la présence d'adverbes de discours dont on a un très bel exemple au folio $42 \mathrm{v}^{\circ}$ : "Décidément, je crois que cette promenade à l'île ne sera que quand je m'amuse déjà avec Albertine. » L'adverbe renvoie en effet au dialogue que tisse l'écrivain entre lui et sa propre production et fonctionne comme une conclusion intérieure, mais dont les traces sont rendues visibles sur le papier.

Autre témoin de ce dialogue entre l'auteur-scripteur et l'auteur-relecteur, la précision du lexique de l'écriture dans les notes. Au-delà des termes « cahier » et «page », qui sont fréquemment utilisés, Proust renvoie aussi dans l'espace du Cahier à «ce qui vient avant » ou " après », ou encore au folio même avec l'emploi des termes « recto » ou « verso » et projette l'espace de l'œuvre publiée, dactylographiée et composée dans l'espace du cahier de brouillon, en annonçant un « alinéa » $\left(f^{\circ} 80 \mathrm{v}^{\circ}\right.$, voir fig. 5$)$. Si l'écriture des brouillons est labyrinthique, Proust laisse cependant des traces précises pour assurer la relecture de son propre cahier, soit à travers un système de renvois dont il faut d'ailleurs souligner la précision, soit par les notes de régie elles-mêmes :

ce que j'ai barré est au recto en face et Suivre les indications (Cahier 46, $\mathrm{f}^{\circ} 48 \mathrm{r}^{\circ}$ ).

À ces précisions concernant le fil de l'écriture et l'organisation du brouillon lui-même, presque maniaques parfois, lorsque Proust renvoie à une page qu'il situe « 22 pages moins loin » $\left(\mathrm{f}^{\circ} 83 \mathrm{r}^{\circ}\right)$, semble s'opposer l'imprécision qui gouverne le lieu d'insertion de certaines notes ou leur statut dans le roman. Ainsi les mots «quelque part» (fos $62 \mathrm{v}^{\circ}$, fig. 7, $\left.67 \mathrm{v}^{\circ}\right)$, «par ici $»\left(f^{\circ} 99 v^{\circ}\right)$, « ici ou ailleurs » $\left(f^{\circ} 62 v^{\circ}\right)$ ou « à un endroit $»\left(\mathrm{f}^{\circ} 78 \mathrm{v}^{\circ}\right)$ viennent souligner les hésitations de l'auteur. De même que les «sans doute » $\left(\mathrm{f}^{\circ} 62 \mathrm{v}^{\circ}\right)$ et les très fréquents «peut'être » (une vingtaine) qui jalonnent le cahier, les modalisateurs, «je crois» comme « je pourrais », apparaissent régulièrement ${ }^{41}$. Ces deux aspects du style des notes - précisions et hésitations - s'ils pourraient apparaître comme contradictoires, témoignent cependant tous deux d'une des caractéristiques principales des notes de Proust, le dialogue constant de l'auteur par rapport à son propre texte. Si Proust est si précis, c'est qu'il entend être en capacité de se relire. S'il hésite, c'est là encore une forme d'interrogation de l'auteur sur son propre texte. Dans un cas, la première instance - celle qui écrit en sait plus que la seconde - celle qui relit, corrige et réécrit - parce qu'à l'instant de l'écriture elle détient la mémoire du texte et de son cheminement; dans l'autre, au contraire, elle est supposée en savoir moins que le je relecteur qu'elle projette comme le détenteur d'une connaissance supérieure de l'œuvre. Entre les deux je, c'est l'épaisseur du temps qui s'intercale.

\section{Dialogues intérieurs}

On retrouve ainsi « la dimension interlocutive propre à l'avant-texte 42 »dont Almuth Grésillon a montré l'importance chez Zola. Elle met en effet en évidence le paradoxe que le brouillon, qui est en principe un « écrit pour soi » et donc destiné à n'être lu que par l'écrivain, comporte une dimension interlocutive forte et elle en conclut que ce dialogue est un dialogue de l'auteur avec lui-même, « et plus précisément de l'auteur-scripteur avec l'auteur-lecteur lisant son propre écrit, le commentant,

41. Voir les folios $42 \mathrm{v}^{\circ}, 46 \mathrm{v}^{\circ}, 75 \mathrm{v}^{\circ}$.

42. Voir Almuth Grésillon, « Le langage de l'ébauche : parole intérieure extériorisée », [en ligne], disponible sur le site de l'ITEM, <www.item.ens. fr>, chap. III. 
l'interrogeant, l'évaluant 43 ». Dès lors, on peut voir les notes comme autant d'actes de langage impliquant les deux instances internes à l'auteur - le scripteur et le lecteur. Almuth Grésillon les classe en trois catégories : l'auto-injonction, l'auto-interrogation et l'autoévaluation 44 . Tout comme pour l'avant-texte de La Bête humaine de Zola, ces trois fonctions des notes - s'évaluer, s'interroger et s'enjoindre - sont abondamment illustrées par le cahier. La fonction d'évaluation de la note est très fréquente. Il peut s'agir de commenter le style, comme dans le laconique « dire mieux 45 » régulièrement employé par Proust, ou même l'action et les personnages du futur roman :

Peut'être tout cela mieux pour q. qu'un d'autre car bizarre que le lift m'écrive (Cahier $46, f^{\circ} 62 \mathrm{v}^{\circ}$, voir fig. 7).

On perçoit ici très clairement les traces d'un dialogue intérieur entre l'instance écrivante (qui vient d'écrire une note pour une lettre du lift au narrateur) et l'instance qui lit (pour qui finalement une telle lettre serait curieuse et qui propose de reporter cette note pour un autre personnage). Pour Almuth Grésillon, tous ces énoncés évaluatifs se ramènent finalement à des énoncés performatifs virtuels du type «j'estime que $46 .$. ». En ce qui concerne l'auto-interrogation, il en a déjà été question précédemment car cette structure est fréquente. Chaque phrase interrogative des notes peut en effet être interprétée comme un acte de questionnement du type « je me demande si ». Pour ce qui est de l'autoinjonction, on remarque que, dans toutes les notes commençant par un verbe à l'infinitif comme «mettre » ou « ajouter » déjà évoquées, il s'agit implicitement d'ordonner, et ici ordonner revêt bien un double sens : se donner un ordre et organiser le récit à venir.

Toutes ces formes - auto-injonction, autointerrogation et auto-évaluation - constituent les traces d'un « dédoublement sujet-auteur 47 » et matérialisent le dialogue intérieur. Comme le souligne Almuth Grésillon, « le langage de l'ébauche » est une «parole intérieure extériorisée » :

[...] l'avant-texte est comme une sorte de méditation à haute voix sur la possible « mise en œuvre », un jeu d'hypothèses, d'alternatives, de questions-réponses, d'auto-injonctions et d'évaluations autoréflexives à propos d'un projet d'écriture, une parole de soi à soi, d'où devrait découler un acte, en l'occurrence un acte scriptural ${ }^{48}$.

Le brouillon est orienté vers le faire, vers l'œuvre à faire, ce qui fait de lui un acte performatif. On en revient à la dimension de «projet » des notes, bien soulignée par Anne Herschberg Pierrot ${ }^{49}$. Non seulement, le cahier de brouillon vise l'étape ultime 50 du «Livre51 » ou de l'œuvre achevée et publiée et contient par là sa propre projection - l'œuvre future mais il concentre également en lui-même son propre passé, puisqu'il porte les traces successives de son écriture et de ses relectures.

Les traces de cette dimension interlocutive se lisent clairement dans les brouillons de Proust à travers l'emploi récurrent du verbe "dire »: « dire mieux toujours » (paperole collée au-dessus du folio 52.2), « dire aussi (capital) » $\left(\mathrm{f}^{\circ} 57 \mathrm{r}^{\circ}\right)$, « cela expliquera sans le dire les descriptions » $\left(f^{\circ} 57 \mathrm{r}^{\circ}\right)$, « il vaut mieux dire » $\left(\mathrm{f}^{\circ} 62 \mathrm{v}^{\circ}\right.$, fig. 7), « dire que » (fos $\left.63 \mathrm{v}^{\circ}, 89 \mathrm{v}^{\circ}\right)$, « dire $\mathrm{q}^{\mathrm{d}}$ je suis jaloux » $\left(\mathrm{f}^{\circ} 66 \mathrm{v}^{\circ}\right)$, « (C'est pour montrer q. q un qui ne répond pas mais ne pas le dire) » $\left(\mathrm{f}^{\circ} 78 \mathrm{v}^{\circ}\right)$, « dire cela mieux » $\left(\mathrm{f}^{\circ} 93 \mathrm{r}^{\circ}\right)$, « bien dire » (fos $95 \mathrm{r}^{\circ}$ ou $94 \mathrm{r}^{\circ}$, voir fig. 6), « dire cela autrement » (f० $\left.96 \mathrm{r}^{\circ}\right)$. En revanche, le terme « écrire », s'il apparaît très souvent dans le texte pour désigner une activité très fréquente des personnages du roman - écrire des lettres - ou pour évoquer l'acte créateur d'un écrivain ou d'un artiste, n'est pas employé par Proust dans ses notes pour désigner sa propre activité d'écrivain. Il est frappant de constater que les deux seuls emplois du verbe dans le Cahier 46 figurent au folio $65 \mathrm{r}^{\circ}$ : « copier ce qui est déjà écrit », «phrase

\footnotetext{
43. Ibid.

44. Ibid., chap. V.

45. Voir par exemple la paperole du folio 52.2 ou le folio $93 \mathrm{r}^{\circ}$.

46. Almuth Grésillon, art. cité, chap. V. 3.

47. Ibid., chap. V.

48. Ibid., chap. I.

49. Anne Herschberg Pierrot, « Les notes de Proust », art. cité, p. 75.

50. Voir Eugène Nicole, art. cité, p. 65.

51. Voir les notes du Cahier 57 relevées par Anne Herschberg Pierrot, qui commencent par «pour le Livre ». «Les notes de Proust», art. cité, p. 77.
} 


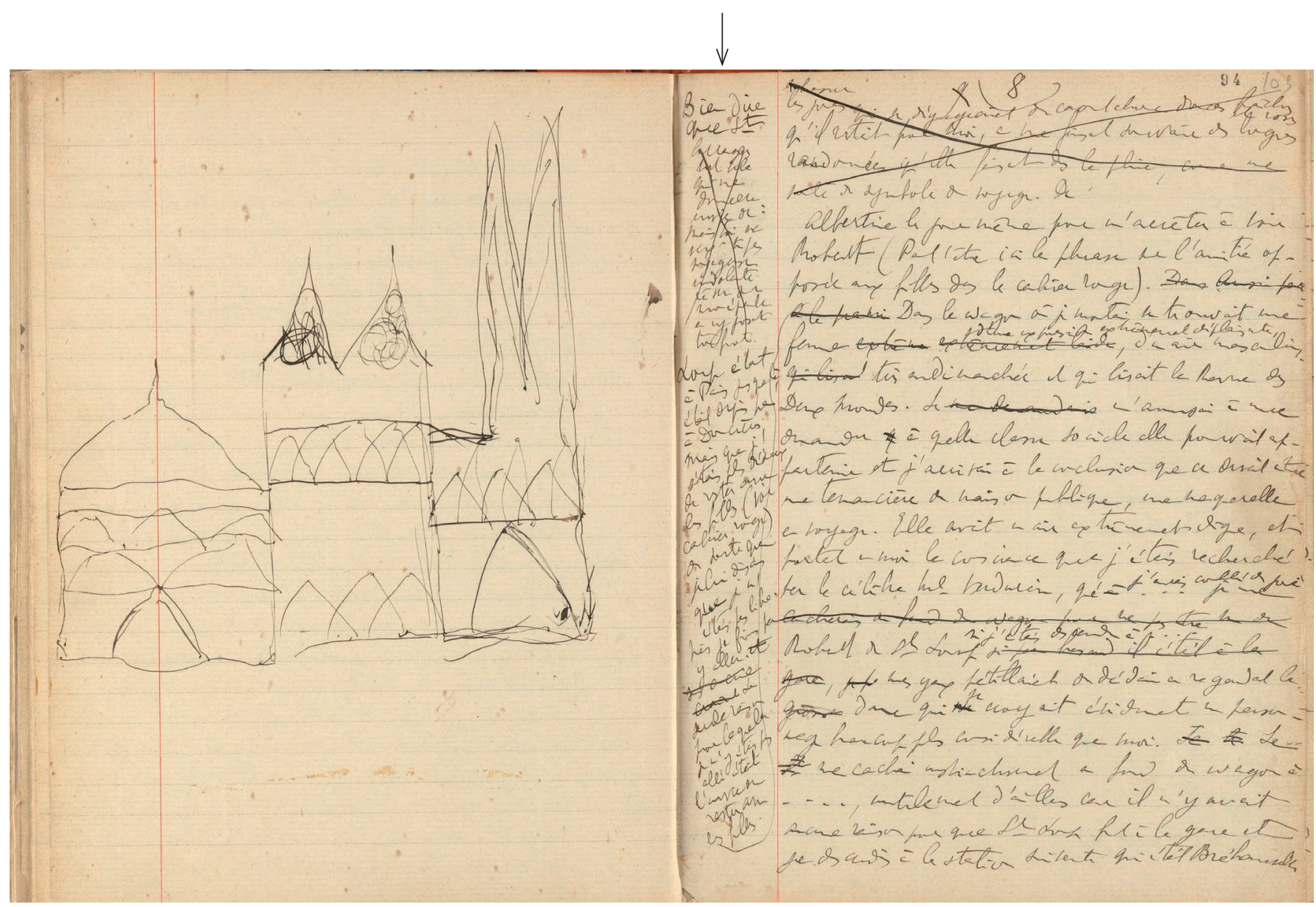

Fig. 6 : Marcel Proust, Cahier 46 (BnF, NAF 16686, fos $\left.93 v^{\circ}-94 r^{\circ}\right)$

écrite ». Il semble donc que pour Proust le texte qu'il est en train d'écrire soit perçu davantage comme une mise en mots sous la forme de parole que de texte. Curieusement, écrire se réfère au passé, à la création achevée, et l'écriture au présent se vit comme l'écho d'une voix. De manière paradoxale, elle se représente elle-même presque plus comme un acte oral que scriptural.

\section{Dialogue interne}

Le dialogue inhérent au brouillon de Proust participe au brouillage des catégories, par exemple ici, en ce qui concerne les catégories de l'espace et du temps :

\section{Je reprendrai ce que $\mathrm{j}$ 'interromps} ici. Mais en ce moment je continue sans plus m' occuper de cette page et $1 / 2$ et Donc ce qui est ci dessous est la suite de 2 pages moins loin au verso

où, comme grâce à quelque pédale, aurait pu survivre la tonalité du bonheur (Non ceci vient avant (Cahier 46, $\mathrm{f}^{\circ} 84 \mathrm{r}^{\circ}$ ).

Présent et futur sont en constante interpolation. L'espace du cahier est un espace labyrinthique avec pour seul fil les notes de l'auteur qui elles-mêmes s'entrecroisent. Le spatial et le temporel échangent même leurs signes puisque avant/après et loin/ci-dessous en viennent à désigner une même réalité. 
Au brouillage des catégories temporelles et spatiales, il faut ajouter également la confusion des notes et des additions. En effet, les catégories qu'on a évoquées se trouvent sans cesse remises en cause par le brouillon lui-même. Au folio $62 \mathrm{v}^{\circ}$, par exemple (voir fig. 7), le passage commence comme une note :

N. B Ajouter Capital au recto précédent. Il vaut mieux dire : « il faut absolument que vous la rameniez » la fois (toujours au même recto où il ne la ramène pas. Ce sera un peu de sa faute, ith

ai pas pris le bon

« je/j’ me suis trompé de chemin, ça m'a un peu allongé elle venait de partir ». Il sentait que cela allait me le (sic) fâcher, aussi le dit-il en ricanant non pa[rce] qu'il fût méchant mais parcequ'il (sic) était timide et croyait diminuer l'importance de sa faute en la traitant en plaisanterie (Cahier 46, $\left.f^{\circ} 62 v^{\circ}\right)^{52}$.

Proust utilise le fameux «capital », souligné, et l'infinitif. Les temps majoritaires sont le présent et le futur. Mais l'introduction du discours direct entraîne un changement de l'énoncé : le discours devient récit, d'où l'emploi final de l'imparfait et du passé simple. Proust lie, dans un même mouvement d'écriture, la note à l'addition.

Et le procédé gouverne le cahier tout entier, notes et additions sont très souvent inséparables et la rédaction elle-même peut prendre naissance dans la note et se fondre en elle 53 . Au dialogue intérieur s'ajoute donc un dialogue interne qui s'effectue cette fois entre la note et le texte. Ainsi, on trouve au bas du folio $95 \mathrm{v}^{\circ}$ (voir fig. 2) ce qui commence comme une note :

Il vaudra peut'être mieux que le retour ensemble par les prairies n'ait pas lieu le

Puis l'écriture se poursuit dans la marge en deux parties :

même jour,

ou bien, je

dirai ttu dès

que j'eus compris

que même Al-

bertine l'eut-

elle voulu

\author{
elle ne pourrait \\ plus + \\ $+$ \\ rencontrer \\ St Loup, je \\ fus calme. \\ Ainsi à \\ peine le \\ train fut-il \\ reparti quand \\ elle retomba \\ dans mes bras, ma \\ colère était passée \\ et alors je pourrai \\ dire voyageuse \\ indolente (Cahier 46, $\left.\mathrm{f}^{\circ} 95 \mathrm{v}^{\circ}\right)$.
}

Le futur signale encore le style notatif au début du passage, mais très vite, le récit s'imbrique dans le discours avec l'emploi du passé simple et du passé antérieur. La dernière phrase met en évidence la fusion des deux types d'énoncé et la confusion entre note et texte, la transition s'effectuant par un simple mot de coordination : « et ». Il faut noter également l'absence de démarcation entre « je pourrai dire 54 » et « voyageuse indolente » qui n'appartiennent pas au même niveau de discours. Proust intègre à sa prose les mots de Vigny dans le poème des Destinées, « La Maison du berger 55 », introduisant du même coup un autre dialogue : celui qui s'établit avec les discours antérieurs 56 .

52. Mise en page simplifiée.

53. Eugène Nicole a lui aussi souligné cet aspect des brouillons de Proust. Il écrit qu' « il y a là sans doute un fait curieux. Curieux parce qu'il s'oppose à notre conception du système notatif comme démarqué du texte du brouillon, à la fois dans sa forme et dans sa position. Troublant même, puisque l'écriture nous apparaît pour ainsi dire interrompue par un élément qui n'est pas de même nature », art. cité, p. 64.

54. Voir également un autre bon exemple de cette pratique au folio $94 \mathrm{v}^{\circ}$. 55. Voir l'avant-dernière strophe du poème de Vigny : « Mais toi, ne veux-tu pas, voyageuse indolente, / Rêver sur mon épaule, en y posant ton front? »

56. C'est une autre dimension du «principe dialogique » qui apparaît ici, souvent commentée, sa dimension intertextuelle. 


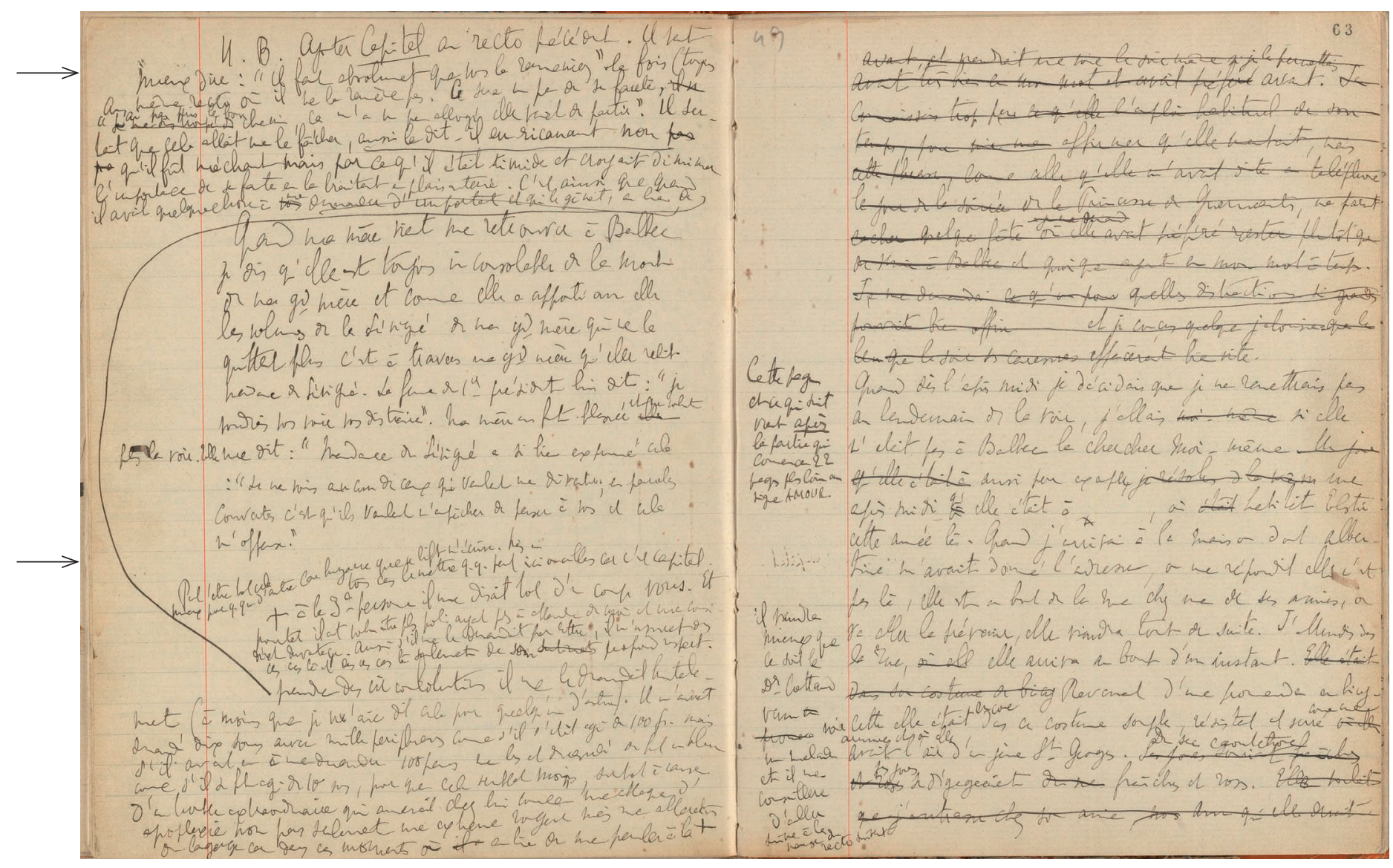

Fig. 7 : Marcel Proust, Cahier 46 (BnF, NAF 16686, fos $\left.62 v^{\circ}-63 r^{\circ}\right)$

De même, au folio $79 \mathrm{v}^{\circ}$ (voir fig. 3), le récit l'emporte progressivement sur la note :

Dans les temps qui suivront qd je 57 stris de temps en temps j'accompagne Albertine chez sa tante à .... (petite ville genre Villerville) ${ }^{58}$.

Sans doute la ceinture d'occupations qui fa entouraient Albertine n'était plus aussi mystérieuse pour moi, mais elle avait un charme d'un autre genre. Anxieux quand elle 59 Albertine me disait : « Non il faut que j'aille chez ma tante 60 de ce qu'elle pouvait bien aller faire, si elle me disait : « Mais pourquoi ne viendriez vous pas chez ma tante, alors tout d'un coup cette fin de journée si anxieuse se finissait dans ce calme divin des fins d'après-midi d'où un orage a été écarté. Et dans le petit salon de la villa donnant sur la salle à manger par une porte à coulisse qu'on faisait glisser dans ce moment même où j'avais des larmes de reconnaissance qu'on gard reçût, qu'on gardât le paria, je sentais bien que j'étais au contraire pour eux le héros de la fête. Tout mon idéal eût été qu'Albertine ne quittât jamais Villerville, pouvoir y posséder une villa attenante à la sienne ne jamais la quitter. Mais puisque ce ne pouvait être ainsi je tâchais de ne pas la voir trop souvent, pour ne pas avoir à recevoir trop souvent le choc de : «Demain je fais telle chose », pour ne pas avoir à obtenir d'elle trop souvent le calmant divin mais qu'elle n'eût peut'être pas indéfiniment multiplié de la ramener chez sa tante (peut'être petit milieu politique ; peut'être même ce que je lui dis chez Me Swann là, et cela me plaît ainsił, Me Cottard pourrait y venir) » (Cahier 46, fo $\left.79 v^{\circ}\right)^{61}$.

57. « Je » devrait être barré.

58. Les noms de lieux ne sont pas encore tous fixés.

59. Proust a répété le sujet : « elle », «Albertine ».

60. Les guillemets ne sont pas refermés.

61. Mise en page simplifiée. 
À la fin du passage, cependant, c'est le style de la note qui envahit de nouveau l'écriture en dessinant les virtualités possibles du roman : définition des personnages, intégration dans le récit, rapport avec les autres personnages du roman. Comme dans les carnets, les passages du métalangage au langage sont incessants dans les cahiers ${ }^{62}$. Si la note, de par sa position marginale au bord de la page ou du folio, surgit du récit, elle se transforme aisément en addition en développant les mots de la page pour tout aussi vite donner naissance à un nouvel objet d'écriture. C'est dans ce dialogue entre l'écriture marginale et le texte qu'on peut lire le processus de la création littéraire, c'est lui qui la nourrit. Et il s'agit ici également d'une esthétique de la Recherche qui ne fait pas de distinction entre la théorie sur le roman et le roman.

La définition de la note comme écriture marginale et autonome se trouve donc remise en cause par la pratique même d'une écriture qui, faisant fi des contraintes de l'espace, redessine son propre lieu, entremêle note et addition et se vit comme un langage intime sur le mode du dialogue. Elle se révèle essentiellement dialogique parce qu'elle est issue d'un double dialogue : le dialogue entre la marge et le texte, le dialogue entre l'auteurscripteur et l'auteur-lecteur. Au terme de cette étude, trois éléments me semblent essentiels en ce qui concerne cette pratique des notes chez Proust : le dialogue des notes avec le texte mais aussi de l'auteur avec lui-même ainsi que la représentation orale de l'acte même d'écrire. Comment comprendre ce qui lie ces trois aspects des notes ? Ce que ces notes révèlent, c'est une conception essentiellement dialogique de l'écriture chez Proust. Elle n'est pas enfermement dans la chambre de liège et repli sur soi : elle est dialogue - dialogue de soi à soi, dialogue avec l'autre, dialogue du texte avec le texte, dialogue enfin avec les autres textes. C'est donc sa dimension interlocutive qu'il faudrait souligner ainsi que la présence d'une altérité qu'elle dessine en creux, que celle-ci soit intérieure, interne ou extérieure.

Faut-il y voir aussi la trace de cette « conversation avec maman » qui occupait dans le projet initial du roman qu'on a appelé le «Contre Sainte-Beuve » une place primordiale ? En tout cas, l'écriture chez Proust se représente comme un dialogue avec lui-même autant qu'un dialogue avec son œuvre et avec les autres œuvres. Le brouillon est ce lieu intime où l'écrivain s'écrit en même temps qu'il écrit, se dit en même temps qu'il dit. Serait-ce à dire que chez Proust aussi « l'être humain [...] n'existe qu'en dialogue 63 » du fait de son hétérogénéité ? La dimension dialogique des notes n'informe pas seulement une pratique, elle dévoile et découvre en même temps la pluralité des « moi » et des êtres 64 .

62. Antoine Compagnon, « Disproportion de Proust [...] », art. cité,p. 163. Anne Herschberg Pierrot le souligne également : «Un trait majeur de l'écriture proustienne est bien l'amplification par l'ajout du commentaire, et l'intégration du métalangage énonciatif à l'énoncé ». Pierre-Marc de Biasi, Anne Herschberg Pierrot, Jacques Neefs, « Ajout et genèse », art. cité, p. 38-39.

63. Voir Tzvetan Todorov, Mikhaël Bakhtine, op. cit., p. 9.

64. D'ailleurs, on sait que chez Bakhtine la théorie littéraire recoupe et recouvre une anthropologie. Voir le chapitre « Anthropologie philosophique », ibid., p. 145-172. 
Julie André est Docteur en littérature et civilisation françaises de l'université de Paris III et chargée de cours à Sciences Po Paris. Elle poursuit ses recherches littéraires et génétiques sur Proust au sein de l'ITEM. Sa thèse, effectuée sous la direction de Pierre-Louis Rey, est consacrée à la transcription et à l'interprétation de l'un des cahiers de brouillon de Proust, le Cahier 46, dont elle prépare également l'édition dans le cadre de la publication des Cahiers de brouillon de Proust (BnF/Brepols).

Julie André, julie.andre@sciences-po.org

\section{Les notes de Proust, une écriture dialogique . L'exemple du Cahier 46}

Le dialogisme du roman moderne a souvent été souligné depuis les travaux de Mikhaïl Bakhtine et de Julia Kristeva. Or, dans les brouillons de Proust, celui-ci est redoublé. Au dialogue intérieur qui révèle les interrogations incessantes de l'auteur sur son propre texte s'ajoute le dialogue interne à la page, entre l'écriture marginale et le récit, entre ce qui est écrit et ce qui reste à écrire. De la pratique de l'écriture au brouillon de Proust, on retiendra principalement ici les notes de régie, lieu du croisement des différentes voix, pour étudier leur inscription dans l'espace mais surtout leur langage et ses modalisations. L'enjeu est de voir dans ce dialogue aux multiples dimensions présent dans les notes, l'une des sources d'une écriture qui, l'œuvre une fois achevée, jouera sur les instances narratives et ne séparera pas le propos sur l'œuvre de l'œuvre elle-même.

The modern novel's dialogism has often been pointed out since the publication of Mikhail Bakhtin's and Julia Kristeva's works. Now this aspect is intensified in Proust's drafts. The inner dialog that reveals the writer's incessant questioning about his own text, is echoed by the page's internal dialog, between marginal writing and narrative, between what is already written and what is still to be written. Of Proust's writing practice in his drafts we will focus mainly on the "notes de régie" (production notes), where different voices intersect, in order to study their spatial inscription and more importantly their language and modes. The issue is to find in this multidimensional dialog present in the notes one of the sources of a writing which, once the work is completed, will play on the narrative instances and will not dissociate the discourse about the work from the work itself.

Der Dialogismus des modernen Romans wurde seit den Arbeiten von Michail Bachtin und Julia Kristeva oftmals herausgestellt. Nun ist dieser in den Entwürfen Prousts noch sehr viel ausgeprägter. Dem internen Dialog, der das ständige Befragen des Autors über seinen eigenen Text offenbart, fügt sich ein interner Dialog auf der Seite hinzu, zwischen Randbemerkung und Erzählung, zwischen schon Geschriebenem und noch zu Schreibendem. Von der Arbeitsweise Prousts in den Entwürfen wird man hier hauptsächlich die Regieanweisungen berücksichtigen, der Ort, an dem sich die verschiedenen Stimmen kreuzen, um deren Eingeschriebensein in den Raum, aber vor allem auch ihre Sprache und deren Modalisierung zu analysieren. Herausforderung ist es in diesen, in den Aufzeichnungen erscheinenden, multidimensionalen Dialogen eine der Quellen einer Schrift zu erkennen, die, nach der Vollendung des Werks, in den
Erzählmomenten präsent bleibt, und die Worte über das Werk vom Werk selbst untrennbar erscheinen lässt.

Después de los trabajos de Mijail Bajtín y Julia Kristeva, se ha hablado a menudo del dialogismo de la novela moderna. Éste, en los borradores de Proust, se duplica. Al diálogo interior que revela los interrogantes incesantes del autor acerca de su propio texto, se asocia el diálogo inherente a la página, entre la escritura marginal y el relato, entre lo que está escrito y lo que queda por escribir. De la práctica de la escritura en los borradores de Proust estudiaremos principalmente aquí las notas preparatorias, en las que se entrecruzan voces diferentes, para analizar su inscripción en el espacio y, sobre todo, su lenguaje y su modalización. Lo que se busca es percibir, en ese diálogo de múltiples dimensiones presente en las notas, una de las fuentes de una escritura que, una vez la obra terminada, jugará con las instancias narrativas y no separará la reflexión sobre la obra de la obra misma.

L'elemento dialogico del romanzo moderno è stato spesso sottolineato dopo i lavori di Mikhaïl Bakhtine e Julia Kristeva. Negli appunti di Proust è addirittura raddoppiato. Al dialogo interiore che rivela le domande incessanti dell'autore sul suo proprio testo si aggiunge il dialogo interno alla pagina, tra la scrittura a margine e il racconto, tra ciò che è scritto e ciò che resta da scrivere. Dalla pratica della scrittura agli appunti di Proust, ci si occupa qui principalmente delle note di regia, luogo di incontro di diverse voci, per studiare come si inseriscono nello spazio, ma soprattutto il loro linguaggio e le loro modellizzazioni. Lo scopo è vedere in questo dialogo a più dimensioni una delle fonti di una scrittura che, una volta conclusa l'opera, giocherà sulle istanze narrative e non separerà le intenzioni sull'opera dall'opera stessa.

Muitas vezes se refere o dialogismo do romance moderno, na esteira de Mikhaïl Bakhtine e de Julia Kristeva. O que, nos rascunhos de Proust, é particularmente notado. Ao diálogo interior que revela as contínuas interrogações do autor sobre o seu próprio texto acrescenta-se o diálogo interno à página, entre a escrita marginal e a narrativa, entre o que é escrito e o que falta escrever. Da prática da escrita em rascunho de Proust, são aqui principalmente tidos em conta os apontamentos de orientação, ponto de cruzamento de diferentes vozes, para estudar a sua inscrição no espaço mas sobretudo a sua linguagem e as suas modalizações. $\mathrm{O}$ desafio consiste em ver nesse diálogo de múltiplas dimensões presente nas notas uma das fontes de uma escrita que, a obra uma vez terminada, jogará com as instâncias narrativas e não distinguirá o que é dito sobre a obra da obra ela mesma. 